Article

\title{
Effects of a Crossarm Brace Application on a 275 kV Fiberglass-Reinforced Polymer Crossarm Subjected to a Lightning Impulse
}

\author{
Muhammad Syahmi Abd Rahman 1,*®D, Mohd Zainal Abidin Ab Kadir 1,2(D, \\ Muhamad Safwan Ab-Rahman ${ }^{1}$ (D), Miszaina Osman ${ }^{1}$, Shamsul Fahmi Mohd Nor ${ }^{1}$ (D) \\ and Noorlina Mohd Zainuddin ${ }^{1}$ \\ 1 Institute of Power Engineering, Universiti Tenaga Nasional, Kajang 43000, Malaysia; \\ mzk@upm.edu.my (M.Z.A.A.K.); asafwan@uniten.edu.my (M.S.A.-R.); miszaina@uniten.edu.my (M.O.); \\ shamsul@ewt.com.my (S.F.M.N.); noorlina.zainuddin@uniten.edu.my (N.M.Z.) \\ 2 Centre for Electromagnetic and Lightning Protection Research (CELP), Advanced Lightning, Power and \\ Energy Research (ALPER), Universiti Putra Malaysia, Seri Kembangan 43400, Malaysia \\ * Correspondence: syahmi@uniten.edu.my
}

Received: 12 October 2020; Accepted: 20 November 2020; Published: 27 November 2020

\begin{abstract}
The crossarm is an important component of transmission towers, providing insulation for transmission lines at different voltage ratings. Recently, composite crossarms were widely used as a composite tower component and were found to be the most favorable choice for replacing old wooden crossarms. Owing to the satisfactory pilot operation and multiple sets of testing, fiberglass-reinforced polymer (FRP) composite crossarms have been used in Malaysia in both 132 and $275 \mathrm{kV}$ transmission lines since the late 1990's. Since then, some modifications have been proposed to improve the mechanical performance of the crossarm, in order to ensure the reliability of its performance. In this investigation, the effect of a proposed improvement, achieved by installing a brace for the crossarm, was investigated numerically. A simulation study was conducted, with a consideration of the lightning impulse voltage (LIV) and swing angle exhibited by the crossarm. The potential and electric field (E-Field) distribution were analyzed and are presented in this paper. It was found that the potential distribution and E-Field strength for the crossarm and the surrounding air were greatly affected by the installation of the brace.
\end{abstract}

Keywords: FRP crossarm; FEM; critical flashover voltages; electric field stress; composites

\section{Introduction}

Nowadays, composite crossarms are widely used on transmission lines. The development of composite insulation was made possible thanks to the success of fiberglass production and composite crossarms based on it in the field. The use of composite crossarms not only reduces land uptake for transmission lines, but also sufficiently simplifies the installation, as it can be done from a tower vehicle and allows for a compact transmission line [1]. Compared to the wooden crossarm, the composite crossarm may possibly skip up to two replacement cycles of the wooden crossarm, which usually partake place in about 25 to 40 years, depending on the local environmental condition and the quality of timber. In Malaysia, fiberglass-reinforced polymer (FRP) crossarms have been successfully installed in both 132 and $275 \mathrm{kV}$ lines since the late 1990's [2]. FRP crossarms were first introduced in Malaysia as an alternative to the wooden crossarm due to the shortage of good quality wood for crossarm production. This study is based on a current issue in Malaysia, where existing wooden crossarms on $275 \mathrm{kV}$ transmission lines are being replaced by the proposed FRP crossarm. This hollow square-shape 
FRP crossarm was found to be more favourable compared to an insulating crossarm (FRP core with silicone rubber shed) [2] for the replacement, due to the minimum tower modification needed compared to the rigid assembly of the insulating crossarm.

FRP, as a composite material, is recognized for its light weight, strong, and robust properties. Despite these advantages, FRP is vulnerable to ageing and degradation, which can be related to environmental and electrical stresses [3]. Several studies have revealed that ultraviolet radiation and heat progressively degraded the fiberglass structure, which might cause fiber blooming, delamination, and water infusion, eventually shortening the service life of the composite [4-7]. Meanwhile, a creep study was conducted by [8] to understand the extensive age behavior of FRP laminates and the manufacturing quality. As reported in [9], it is predicted that the stiffness of an FRP composite will be reduced by up to $35 \%$ after a year of loading and the rate will increase up to $100 \%$ after 50 years.

Electrical stresses on a composite crossarm were reviewed in [10], which indicates that some parts of the crossarm experienced an irregular distribution of the electric field along the surface at the triple junction. The magnitude of the electrical field on insulating materials such as FRP should be at a minimum to avoid tracking and erosion due to partial discharges. Figure 1 shows an example of surface tracking developed on a sample of a used crossarm [2].

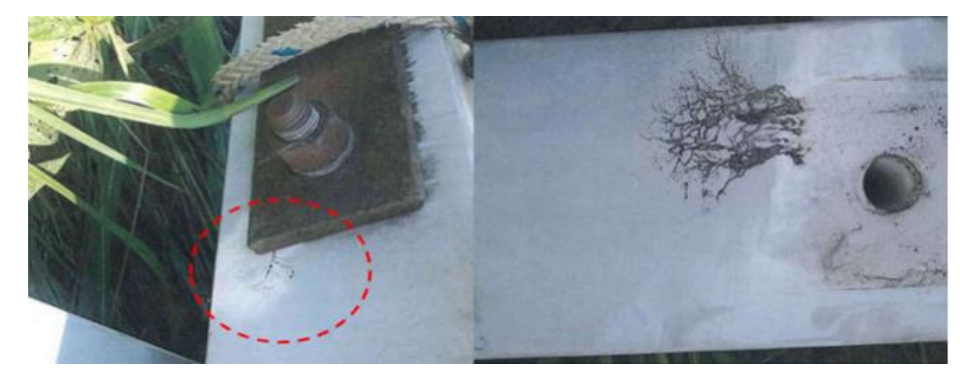

Figure 1. Typical surface tracking on a composite crossarm [2].

The electric field distribution of the FRP crossarm can only be evaluated numerically, for example, by using the Finite Element Method (FEM). Nowadays, FEM has been well-recognized as a convenient and accurate method of computation that can assist in electrical design [11]. It allows the designer to virtually work with the model and review the performance under several conditions before actual high voltage testing, thus saving time and money [12]. FEM analysis has been used for designing electric field stress control devices for insulating crossarms. The component was modeled using FEM and found to work as intended by laboratory testing and successful installations in the field [11]. Meanwhile, in [10], a feasibility study of the inner ground cable of a novel unibody composite crossarm was conducted using FEM, providing an important basis for dismissal of the design before the production of prototypes.

\section{Lightning Overvoltages on the Transmission Line}

In general, the crossarm should be tested under a lightning impulse voltage (LIV) based on IEC 60383-2 Clause 9 and withstand the basic insulation level (BIL) at $1050 \mathrm{kV}$ [13]. The purpose is to verify the insulation strength against the lightning overvoltages that might occur within the overhead lines. The insulation strength of the crossarm and its components can be described by the critical flashover (CFO) voltage. The CFO voltage refers to a $50 \%$ probability of insulation failure when the impulse wave shape with the $\mathrm{CFO}$ peak voltage is applied to the insulation. As an excellent approximation, the CFO of insulation gaps can be derived by the length of the gaps multiplied by a gradient of $560 \mathrm{kV} / \mathrm{m}$ for positive impulse polarity and $605 \mathrm{kV} / \mathrm{m}$ for negative impulse polarity [14,15]. The calculation is as presented in Equation (1).

$$
\mathrm{CFO}=\text { distance } \times \mathrm{CFO}_{\text {gradient }}
$$


Additionally, a numerical study was conducted using commercially available FEM software to evaluate the electrical performance of an FRP crossarm, whereas the potential distribution and magnitude of the electric field were analysed and elaborated. The dimensions of the $275 \mathrm{kV}$ tower used in this study are illustrated in Figure 2.

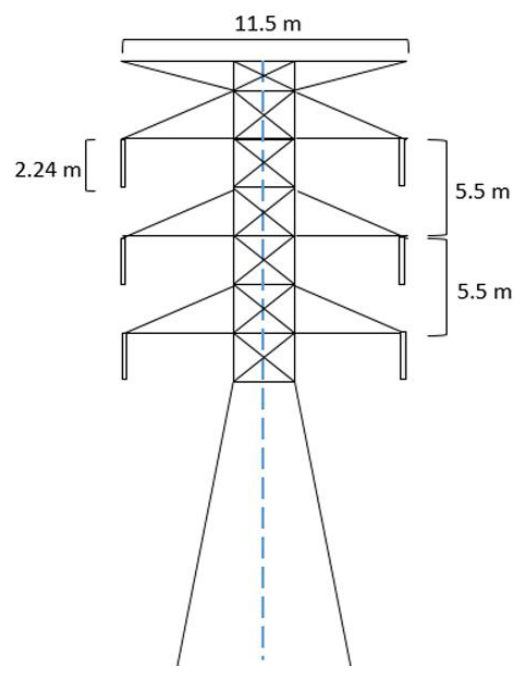

Figure 2. Typical dimensions of a $275 \mathrm{kV}$ transmission line with fiberglass-reinforced polymer (FRP) crossarm.

\section{Application of CrossArm Bracing}

Crossarms were made to withstand the load of the entire span of the phase conductor and the insulators. To some extent, they need to withstand the dynamic forces on transmission line structures, such as vibration, wind, temperature, and mechanical defects such as cable rupture, which can cause chain reaction destruction on all tower assemblies $[16,17]$. It was previously reported that some of the crossarms in Malaysia are experiencing mechanical failure and tend to break during their service. This failure has hassled the power provider, resulting in concerns about the uncontrolled chain reaction that might be initiated. Therefore, an immediate solution to increase the mechanical strength of the existing crossarm by using a brace was proposed. The brace installation requires four additional FRP bars to be attached at the middle span of the crossarm by using steel fittings (refer to Figure 3). This solution is believed to be the most favorable and cost-saving solution that can be applied instead of replacing the crossarms along the affected line. However, the placement of steel fittings in the middle of the crossarm is a concern due to the fact that it might electrically affect the insulation performance, especially in the event of strikes. The additional steel fittings might introduce more areas with localized stress, consequently promoting local discharges on the FRP composites and increasing the risk of damage.

In this case, the steel fittings may act as floating objects between high voltage and ground terminals. According to [18], the insulation strength of the air gaps was significantly reduced due to the presence of floating objects, whereby it varies according to the length of the gaps. Considering this issue, the performance of a crossarm with a brace installed is worth investigating. 


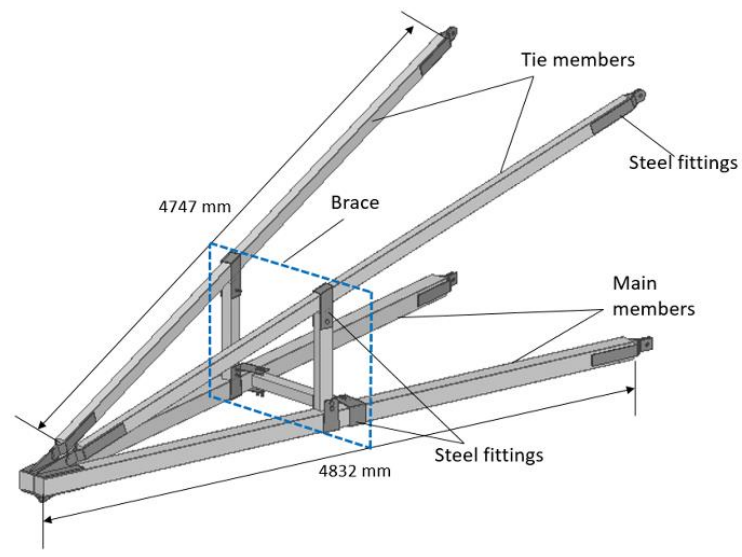

Figure 3. The FRP composite crossarm with an installed brace for the $275 \mathrm{kV}$ transmission line.

\section{CFO Estimation of the Transmission Line}

There were six configurations considered in this study, i.e., crossarms with and without braces at different insulator swing angles. Three angles of $0^{\circ}, 30^{\circ}$, and $57^{\circ}$ were introduced to consider the normal condition, intermediate, and maximum swing, respectively. Distances D1 to D9 were defined to represent the nearest distance phase conductor to the earthed metallic part of the tower (see Figure 4), except for D3, which was defined as the shortest distance to another phase. The estimated CFO values for the given distances were as indicated in Table 1.

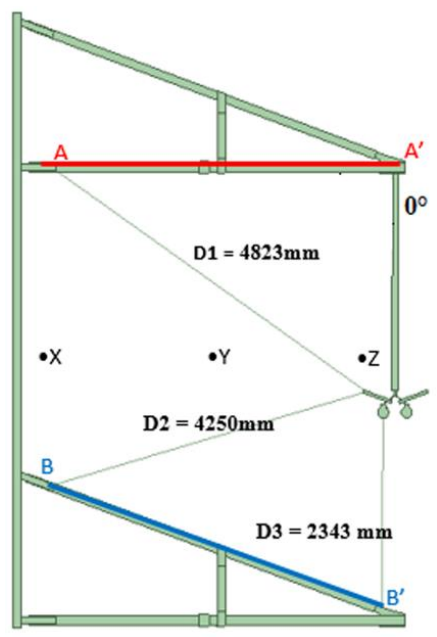

(a)

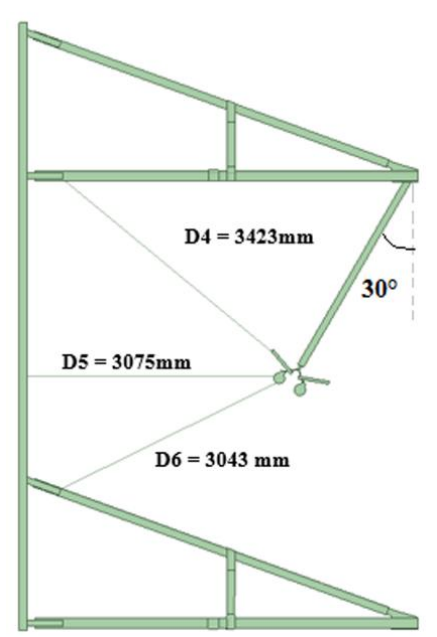

(b)

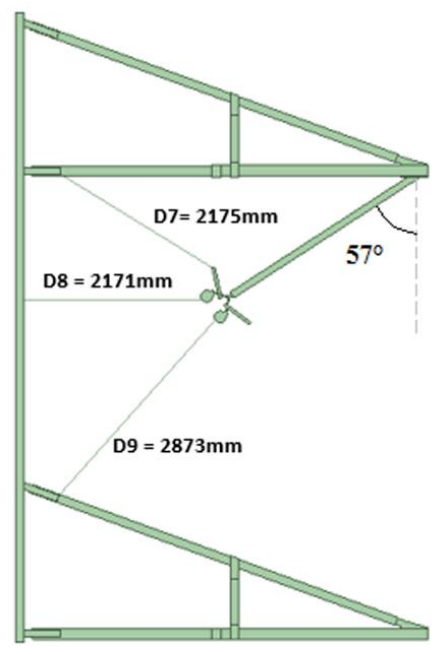

(c)

Figure 4. The distance to the nearest earthed metallic object at different swing angles: (a) Swing angle of $0^{\circ}$; (b) swing angle of $30^{\circ}$; and (c) swing angle of $0^{\circ}$.

Table 1. Estimated critical flashover (CFO) to the nearest earthed metallic object.

\begin{tabular}{cccc}
\hline Length & Distance, $\mathbf{m}$ & $\begin{array}{c}\text { Estimated CFO Corresponds } \\
\text { to + ve LIV, } \mathbf{M V}\end{array}$ & $\begin{array}{c}\text { Estimated CFO Corresponds } \\
\text { to - ve LIV, MV }\end{array}$ \\
\hline D1 & 4.823 & 2.70 & 2.92 \\
D2 & 4.250 & 2.38 & 2.57 \\
D3 & 2.343 & 1.31 & 1.42 \\
D4 & 3.423 & 1.92 & 2.07 \\
D5 & 3.075 & 1.72 & 1.86 \\
D6 & 3.043 & 1.70 & 1.84 \\
D7 & 2.175 & 1.22 & 1.32 \\
D8 & 2.171 & 1.22 & 1.31 \\
D9 & 2.873 & 1.61 & 1.74 \\
\hline
\end{tabular}




\section{Simulation Model}

The numerical approach was conducted by two-dimensional analysis using the commercially available FEM software. The models included a simplified drawing of a crossarm, simplified suspension insulator (of 16 discs), and part of a steel tower. Swing angles were introduced to represent the worst possible case. Each of the objects' materials were based on the parameters given in Table 2.

Table 2. Material-based parameters.

\begin{tabular}{ccc}
\hline Structure & Relative Permittivity $\left(\varepsilon_{\mathbf{r}}\right)$ & Bulk Conductivity $(\boldsymbol{\sigma}), \mathbf{S} / \mathbf{m}$ \\
\hline FRP crossarm & 5 & $1 \times 10^{-16}$ \\
Steelworks/fittings & 1 & $2 \times 10^{6}$ \\
Glass insulator disc & 5.5 & $2 \times 10^{-14}$ \\
\hline
\end{tabular}

A large number of meshing elements were required to discretize the geometry model. Fine meshes were set for the model, particularly in the air and the crossarm. The model was simulated under BIL $(1050 \mathrm{kV})$ to simulate the lightning impulse withstand test. In addition, a shielding failure (SF) induced voltage $(2000 \mathrm{kV})$ was also considered to simulate the condition under such an event. It should be noted that, when a $10 \mathrm{kA}$ direct strike occurs, the waves of current split into halves (5 kA each), which then travel in both directions along the struck conductor. As a result, by assuming a line impedance equal to $400 \Omega$, potential at $2000 \mathrm{kV}$ is generated across the insulation (see Figure 5). In general, the voltages produced during SF on conductors are more significant than those generated during the BF event, so they were considered in this study [19].

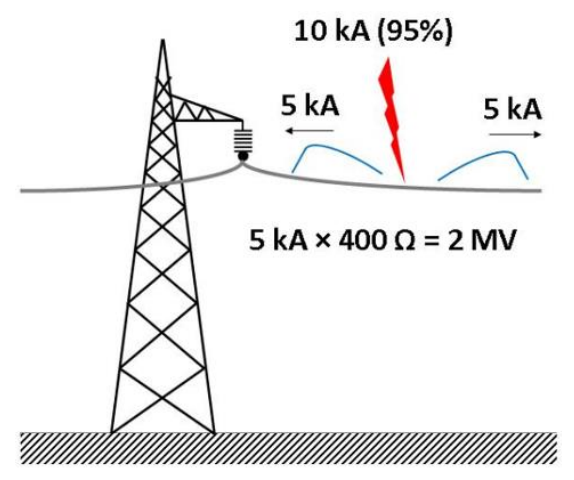

Figure 5. The illustration of a shielding failure event.

The LIV for both BIL and SF were modeled based on a standard waveform $(1.2 / 50 \mu \mathrm{s})$ and double exponential function, as presented in Equation (2):

$$
u(t)=u_{0} k\left(e^{-\alpha t}-e^{-\beta t}\right)
$$

where $\mathrm{u}_{0}$ is the peak value of the lightning impulse voltage, and $\alpha$ is the attenuation coefficient of the wave front, which is set to $1.473 \times 10^{4}$. Additionally, $\beta$ is the attenuation coefficient of the wave tail, which is set to $2.08 \times 10^{6}$, and $\mathrm{k}$ is the correction coefficient, which is equal to 1.043 . The modeled waveform is as illustrated in Figure 6. Later, the waveforms were introduced and applied at the phase conductor for both cases. In the study, the simulations were executed for $100 \mu \mathrm{s}$, with a time step of $0.1 \mu \mathrm{s}$. Table 3 indicates the simulation carried out in the study. 


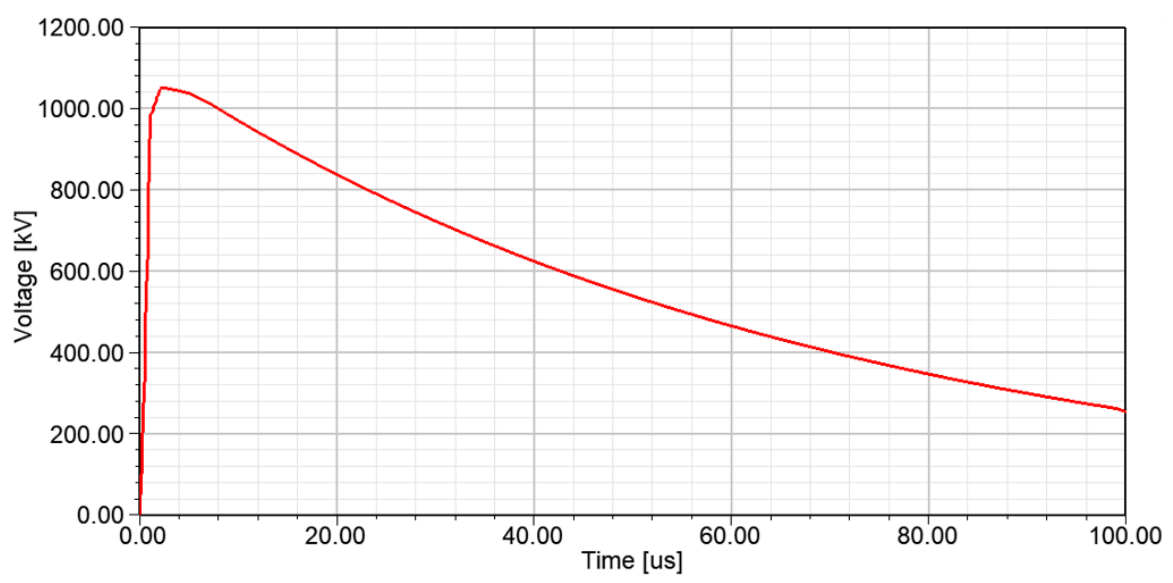

Figure 6. Modeled lightning impulse voltage based on a double exponential function.

Table 3. Simulations conducted.

\begin{tabular}{cccc}
\hline Brace & Swing Angle & Applied LIV (kV) & Type of LIV \\
\hline \multirow{4}{*}{ No } & $0^{\circ}$ & 1050 & BIL \\
& \multirow{2}{*}{$30^{\circ}$} & 2000 & SF \\
& & 1050 & BIL \\
& $57^{\circ}$ & 2000 & SF \\
& \multirow{2}{*}{$0^{\circ}$} & 1050 & BIL \\
& $30^{\circ}$ & 1050 & SF \\
\hline \multirow{2}{*}{ Yes } & $57^{\circ}$ & 2000 & BIL \\
& & 1050 & SF \\
& & 2000 & BIL \\
& & 2000 & SF \\
& & & SF \\
\hline
\end{tabular}

\section{Results}

During the peak moment of the lightning impulse voltage, the crossarm is exposed to the largest voltage and suffers the highest electrical stresses. Therefore, the simulation results presented in this study were projected at the time of the peak, i.e., $1.2 \mu$ s.

\subsection{Effect of Bracing on the Potential Distribution}

During the event of lightning, the potential across the high voltage (HV) and earthed parts abruptly rises to the maximum and gradually diminishes with time. Figure 7 shows the potential measured at points X, Y, and Z when the BIL voltage was applied, and a higher maximum potential can be observed for the case when the brace was installed. The distribution of the voltage during the peak can easily be imagined by referring to the contour plot in Figure 8.

From the contour plot, the highest potential can be seen around the phase conductor, and it gradually decreases towards the earthed tower body. Distinctive potential patterns can be observed in every part of the contour plots due to the propagation of potential in different material. An obvious pattern can be seen in the region across the air and across the crossarm. For the case without a brace, the potential lines are distorted near the crossarm structure. However, a slight change in distribution can be noticed within the circled area in Figure 8, where the higher potential has shifted closer to the earthed parts. It is believed that the conductive properties of the steel brace fittings promoted a path for the traveling voltage. This might have caused a reduction in the insulation strength of the crossarm.

The potential distribution along the distances D1, D2, and D3 for both cases with and without a brace is clearly shown in Figure 9. The maximum and minimum voltage along the distances were recorded, whilst the differences were calculated. 


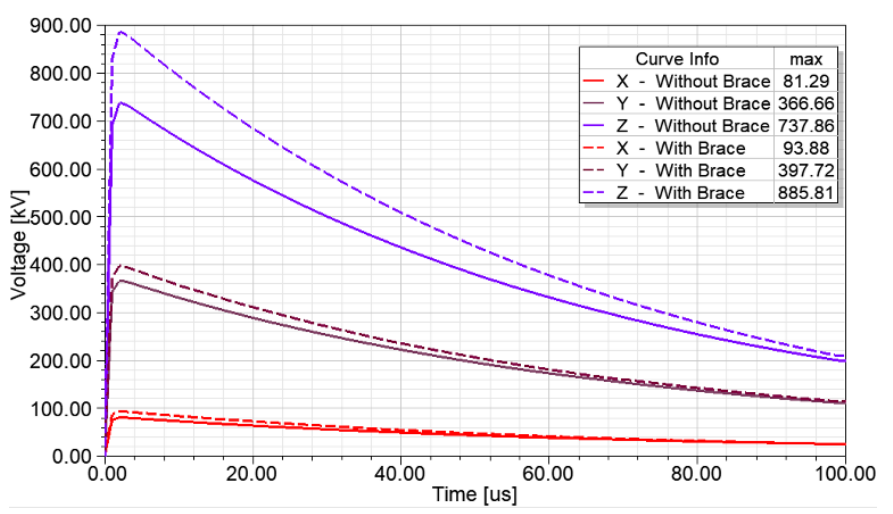

Figure 7. The potential measured at three different points (refer to Figure $4 \mathrm{a}$ ) of the transmission line subjected to basic insulation level (BIL) voltage.

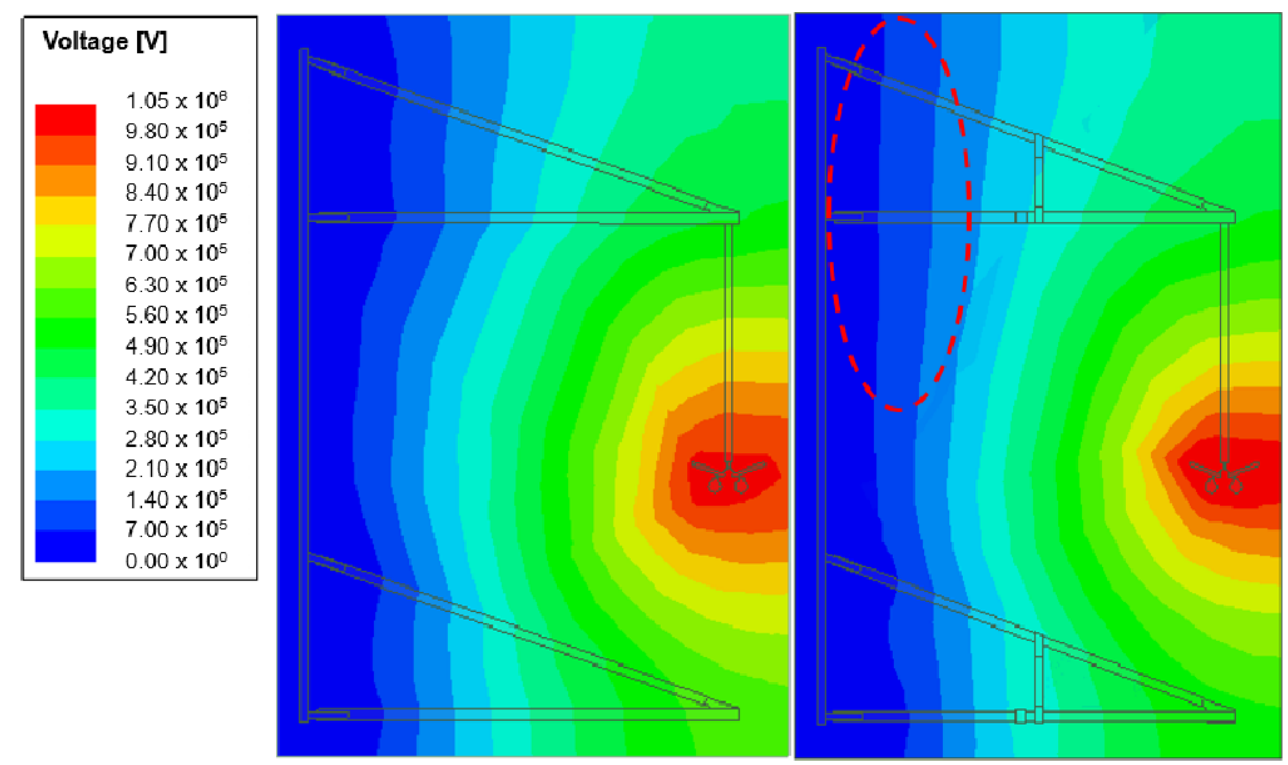

Figure 8. Equal-potential distribution for the case with a non-pivoted insulator under BIL voltage.

Based on Figure 9, none of the voltage stress along the considered distances exceeded the estimated CFO. However, a slight decrease in potential along D1 can be observed to have occurred when the brace was introduced, representing a $4.9 \%$ reduction. Conversely, the potential recorded across D3 indicates a $12.1 \%$ rise. The same trend can be expected for the case of SF, where changes in potential may cause a reduction of the insulation strength, thereby reducing the $\mathrm{CFO}$ voltage by the same fraction. However, the trends were indepedent of the environmental factors that may directly or indirectly influence the breakdown strength of the insulation [20].

In the cases with a pivoted insulator, a different profile of the potential distribution was expected. It can be seen in Figure 10 that the higher voltage region is closer to the earthed tower part than it previously was. Similar to the previous case, the installation of the crossarm brace slightly changed the pattern of distribution near the crossarm end-fittings (circled in red). The potential distribution along the measurement distances D7, D8, and D9 for both cases without and with a brace is presented in Figure 11. The voltage was found to decrease linearly across the measurement lines. A comparison of the two cases revealed a slightly different voltage gradient along D7, causing a reduction of the insulation strength of $10.6 \%$. In comparison, only a minor change of the voltage gradient can be seen at the high voltage end and low voltage end for D8 and D9. 


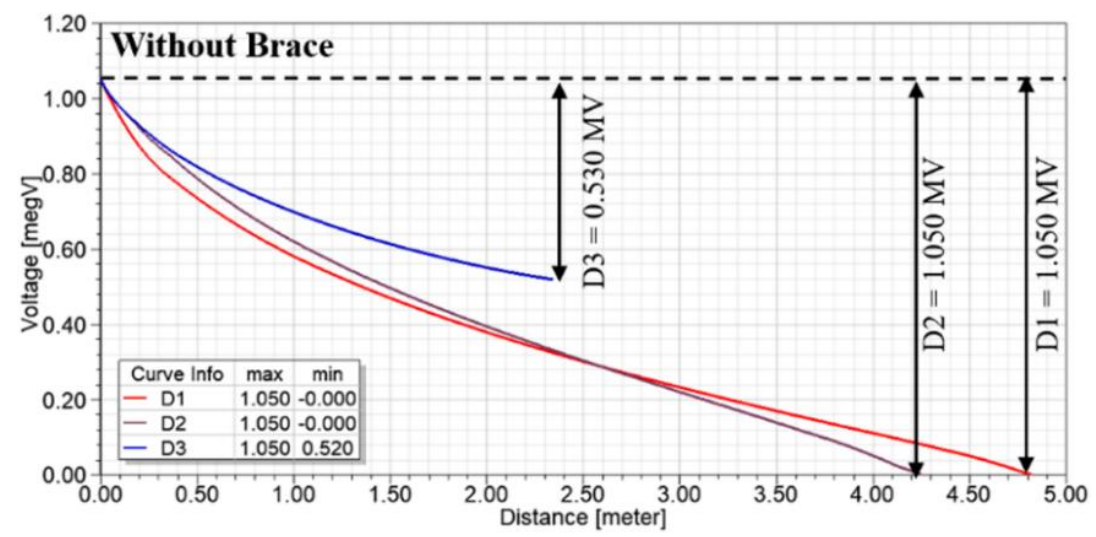

(a)

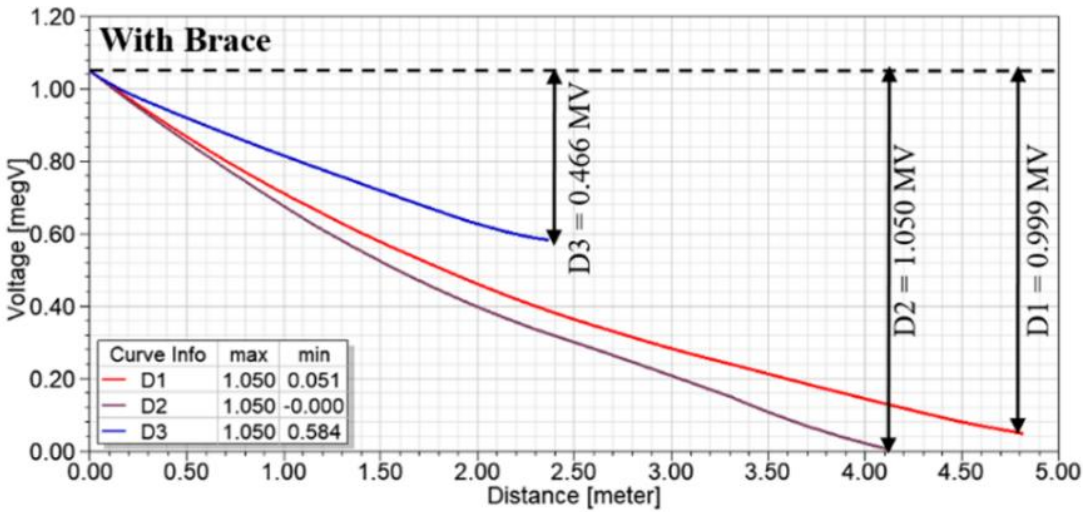

(b)

Figure 9. Potential distribution and the average potential along D1, D2, and D3 for the case (a) without a brace and (b) with a brace.

\begin{tabular}{|l|}
\hline Voltage [V] \\
\\
$1.05 \times 10^{6}$ \\
$9.80 \times 10^{5}$ \\
$9.10 \times 10^{5}$ \\
$8.40 \times 10^{5}$ \\
$7.70 \times 10^{5}$ \\
$7.00 \times 10^{5}$ \\
$6.30 \times 10^{5}$ \\
$5.60 \times 10^{5}$ \\
$4.90 \times 10^{5}$ \\
$4.20 \times 10^{5}$ \\
$3.50 \times 10^{5}$ \\
$2.80 \times 10^{5}$ \\
$2.10 \times 10^{5}$ \\
$1.40 \times 10^{5}$ \\
$7.00 \times 10^{5}$ \\
$0.00 \times 10^{0}$ \\
\hline
\end{tabular}
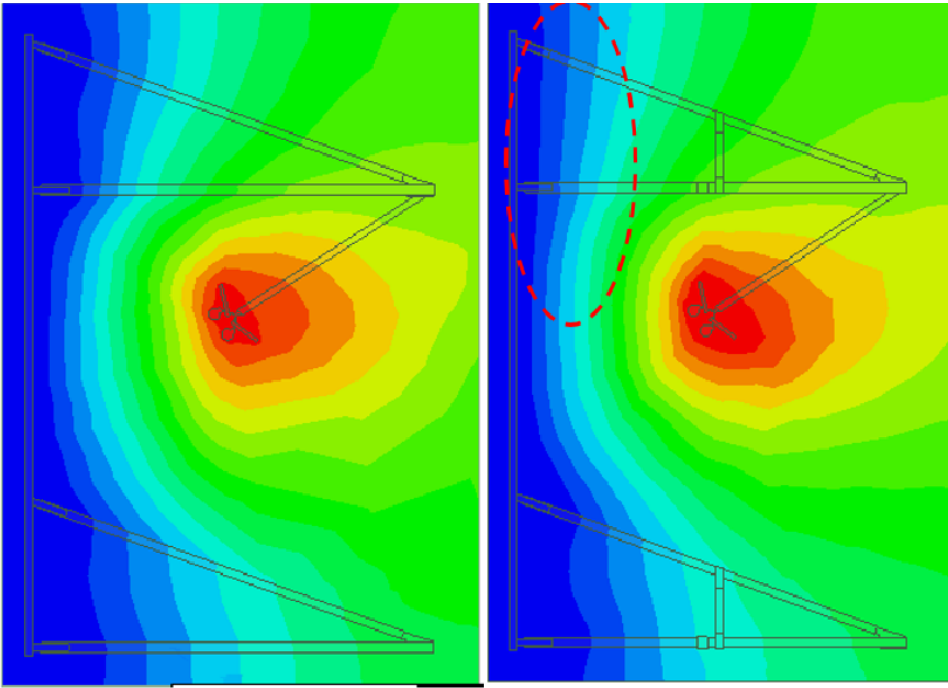

Figure 10. Equal-potential distribution in the case of a $57^{\circ}$ pivoted insulator. 


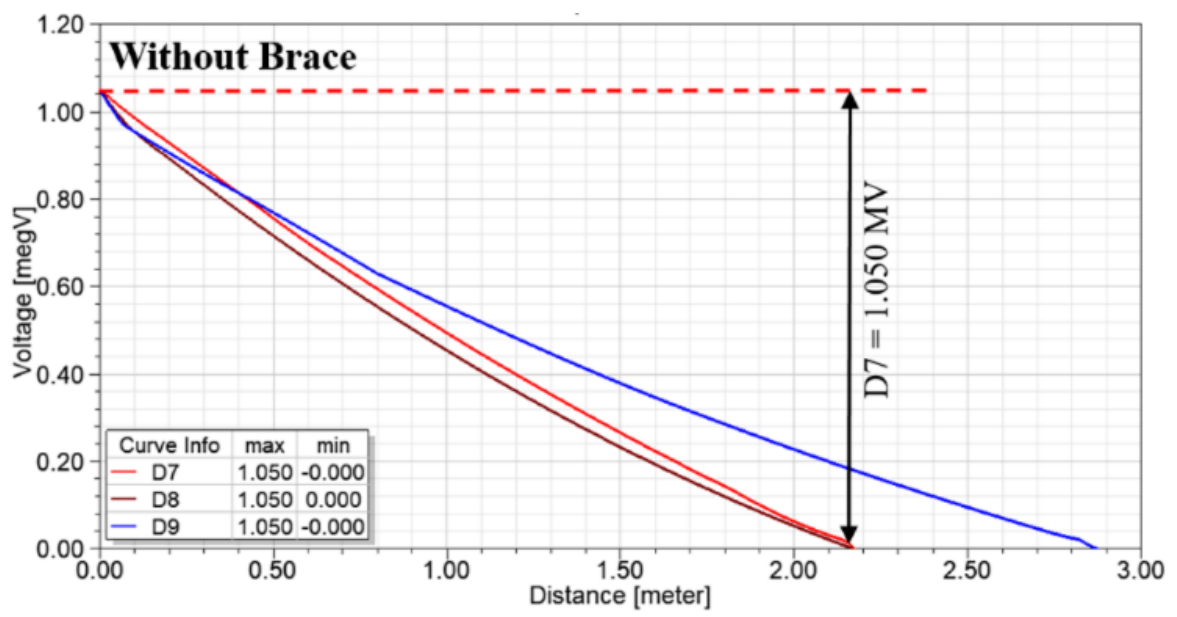

(a)

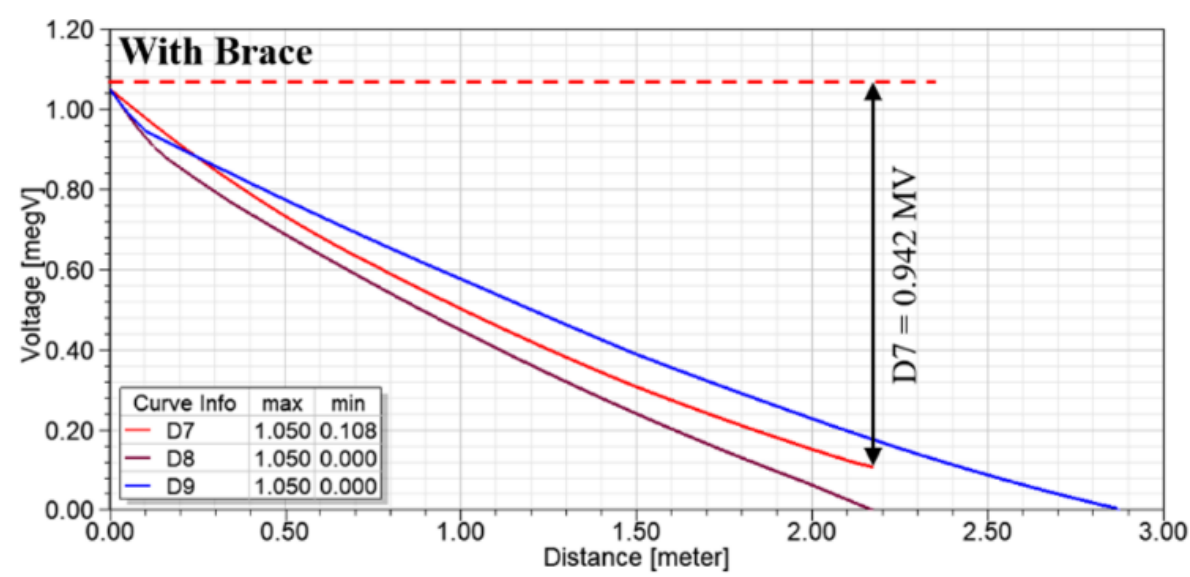

(b)

Figure 11. Potential distribution along D7, D8, and D9 considering a $57^{\circ}$ pivoted insulator for the case (a) without a brace and (b) with a brace.

\subsection{Effect of Bracing on the Localization of E-Fields}

The electric field (E-Field) profiles were associated with the potential gradient per unit distance. A higher potential difference per distance resulted in a higher E-Field stress. It can be seen from the simulation that the region closer to the phase conductor suffers from a higher E-Field intensity. A difference in the E-Field distribution can be obviously seen between the considered conditions (shown in Figure 12). For instance, the installation of a crossarm brace reduced and broadened the area with a high electric field (circled in yellow). In the plots, the maximum E-Field can be found near the phase conductor and its suspension clamp is as high as $1.74 \times 10^{6}$ and $6.11 \times 10^{5} \mathrm{~V} / \mathrm{m}$ for the case without and with a brace, respectively. Normally, this area under a flashover condition is most likely heated and might cause the conductor to melt, as shown in Figure 13 [21]. Perhaps the installation of a brace could reduce the severity.

From Figure 12, it can be seen that the E-Field strength on the FRP crossarm was also influenced by the brace installation. Using FEM-based software allowed for a closer analysis to be conducted on the FRP surface. Measurement lines $\mathrm{A}-\mathrm{A}^{\prime}$ and $\mathrm{B}-\mathrm{B}^{\prime}$ were introduced to measure the E-Field value at the main member and tie member of the alternate crossarm, respectively (see Figure 4a). Localized E-Field can be seen at the low voltage end of the tie member where the triple junction (of the end-fitting, crossarm, and air) occurs. The difference in the E-Field distribution for the case without and with a brace is presented in Figure 14. 


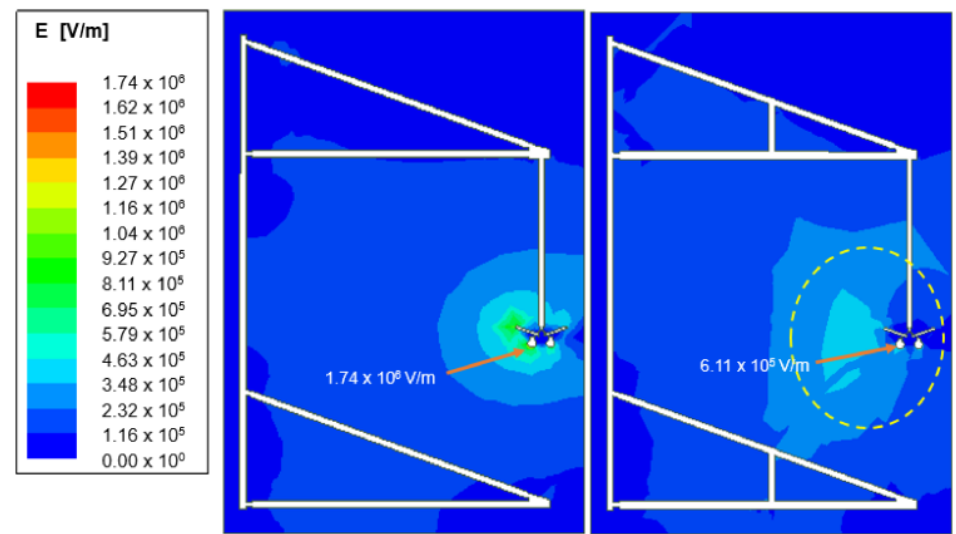

Figure 12. Electric field distribution due to the lightning impulse voltage (BIL).

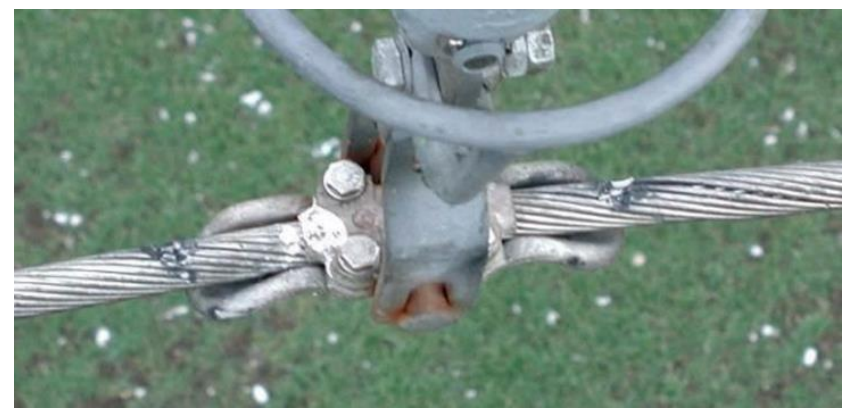

Figure 13. Transmission conductor damaged by lightning [21].
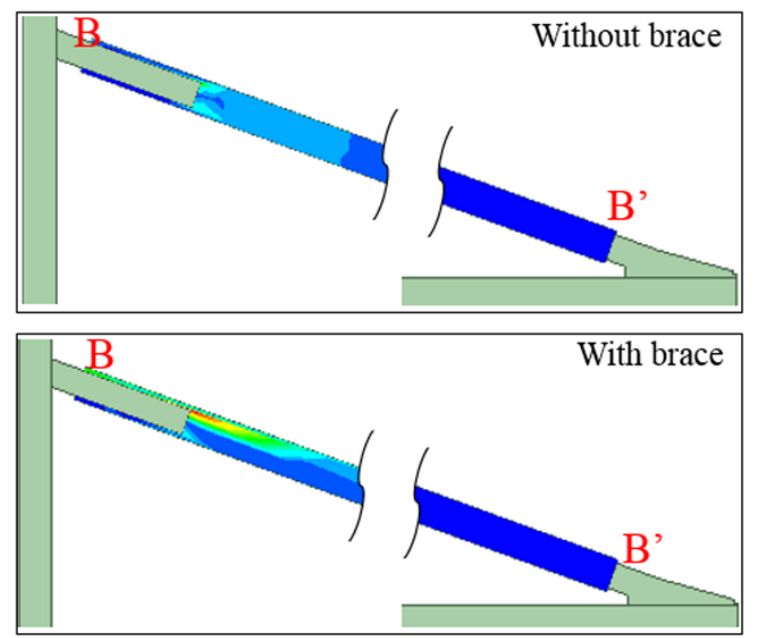

Figure 14. Localized E-Field near to end-fittings of the alternate tie member (B-B').

The EF values measured along B and B' are projected in Figure 15. The installation of the brace caused the stressed area to widen noticeably from 0.16 to $0.55 \mathrm{~m}$, indicating a more than three-fold increase. It can be predicted that the tie member was susceptible to more severe trekking after brace installation. The highest E-Field intensity recorded along B-B' for the case without braces was $6.33 \times 10^{5} \mathrm{~V} / \mathrm{m}$ and $1.25 \times 10^{6} \mathrm{~V} / \mathrm{m}$ for the case with braces, which indicates an increase of stress of almost $97.5 \%$.

On the contrary, a significant reduction of the maximum E-Field can be observed along the main member labeled A-A' (see Figure 16). Figure 17 shows that the brace installation eliminated the E-Field spike near the end-fitting, causing the maximum value of E-Field to be reduced from $1.93 \times 10^{5}$ to $1.46 \times 10^{5} \mathrm{~V} / \mathrm{m}$, which indicates a $24.4 \%$ reduction. Subsequently, the middle part of the crossarm 
experienced a slight increase of the E-Field value that changed the overall profile of the E-Field, indicating a dome-like shape.

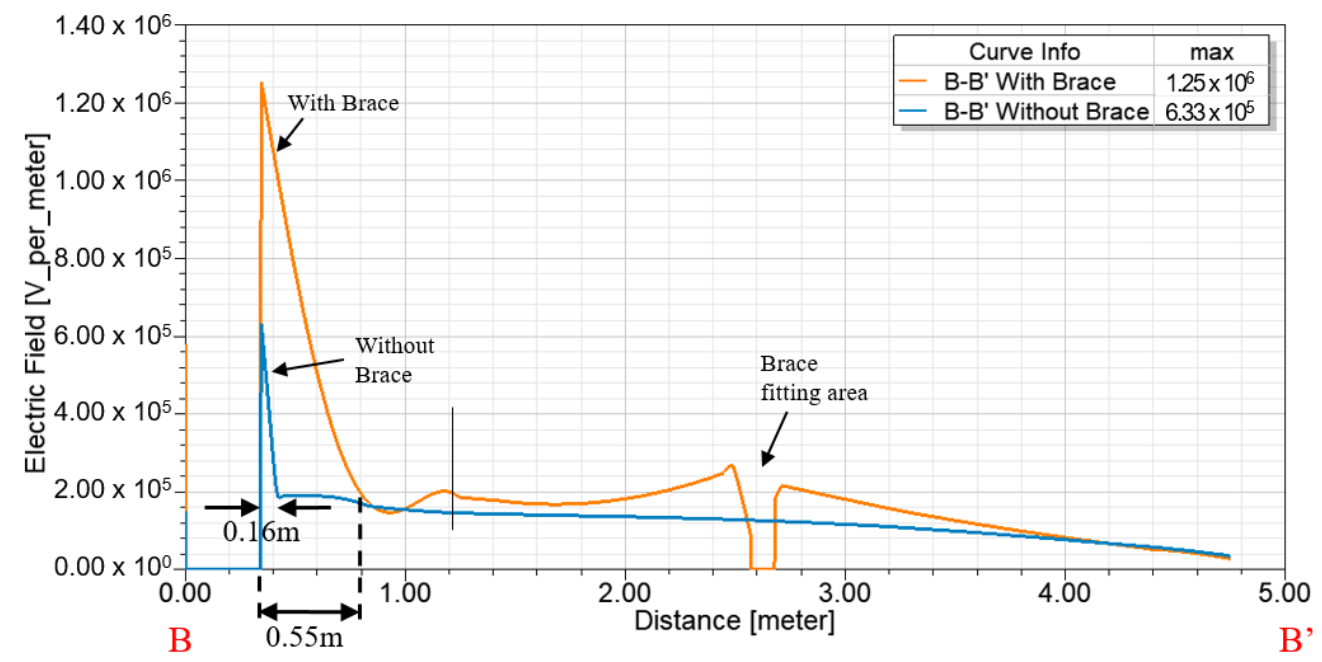

Figure 15. Changes of the E-Field profile and magnitude along B-B' due to brace installation.

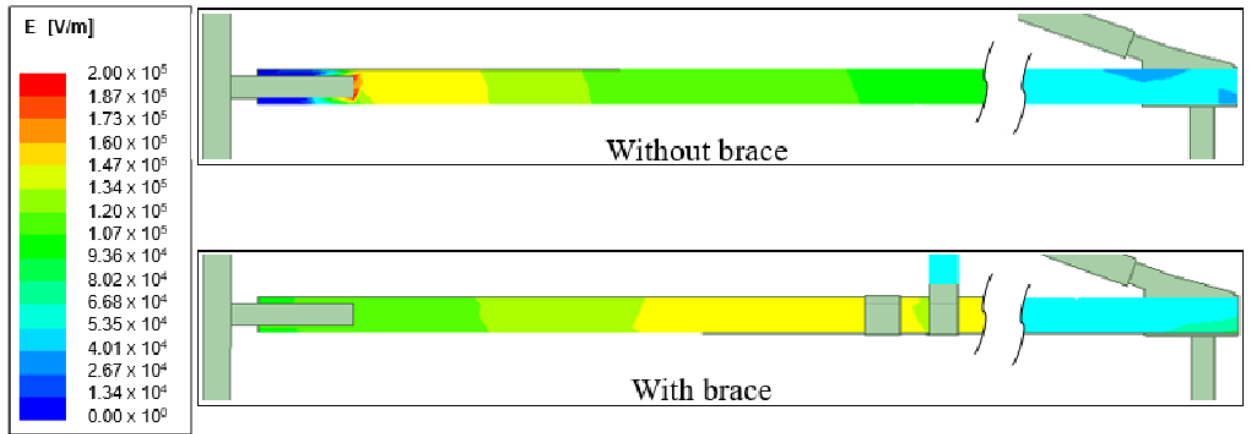

Figure 16. E-Field profiles along the main member (A-A').

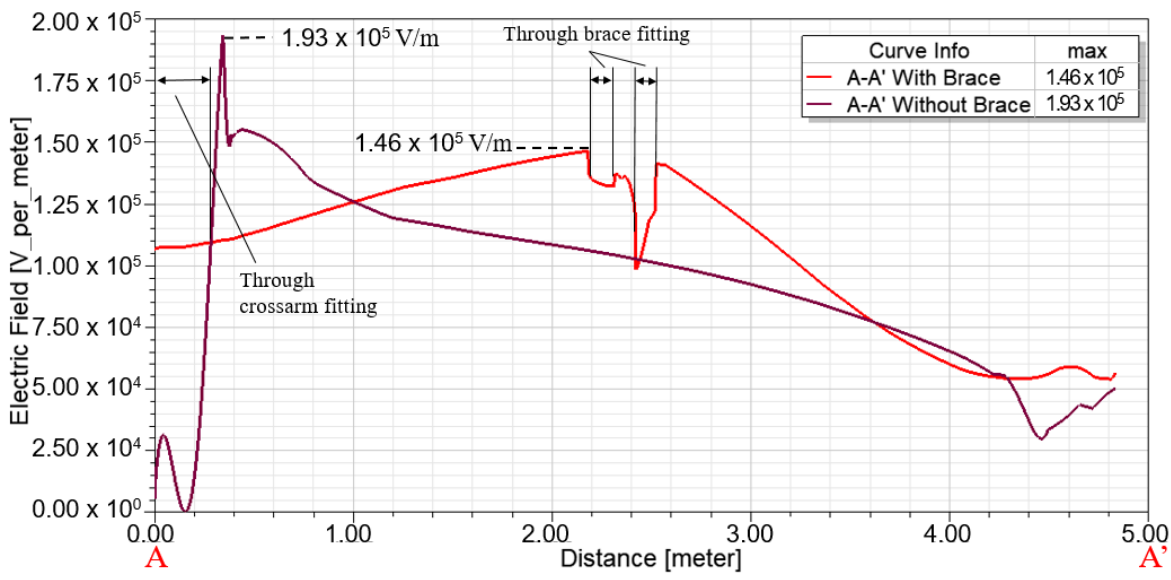

Figure 17. Changes of the E-Field magnitude along A-A' due to brace installation.

\subsection{Consideration of the Swing Effect on the E-Field}

Based on the simulation, the transient computation showed that the E-Field distribution was greatly influenced by the insulator swing. As can be seen in Figure 18, the stresses along the crossarm were found to be reduced when the brace was installed, whilst causing a higher E-Field value to localize around the arcing horn and phase conductor (referred to as air). Figure 19 shows the E-Field 
profile along B-B' in the consideration of swing angles and brace installation. Referring to the E-Field distribution for the non-braced crossarm, the insulator swing of $30^{\circ}$ caused a rise in the maximum E-Field, indicating a $141.7 \%$ increment. However, increasing the swing to $57^{\circ}$ caused a reduction of $22.6 \%$. Meanwhile, for the braced crossarm, a significant reduction of the maximum E-Field of $23.9 \%$ and $29.7 \%$ was recorded for the $30^{\circ}$ and $57^{\circ}$ inclination, respectively.
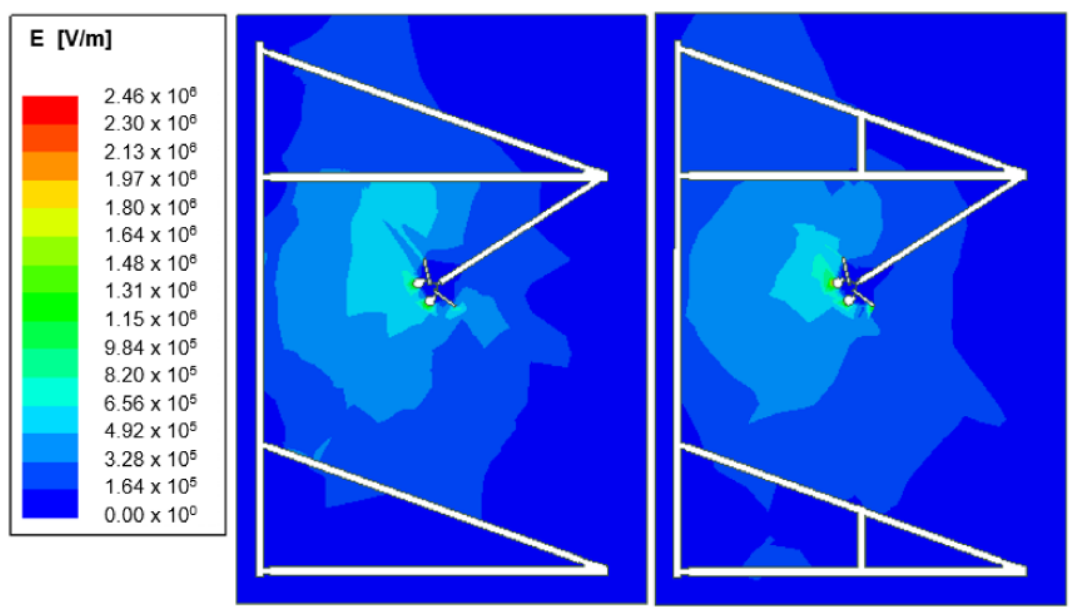

Figure 18. E-Field distribution for the case with a $57^{\circ}$ inclined insulator.

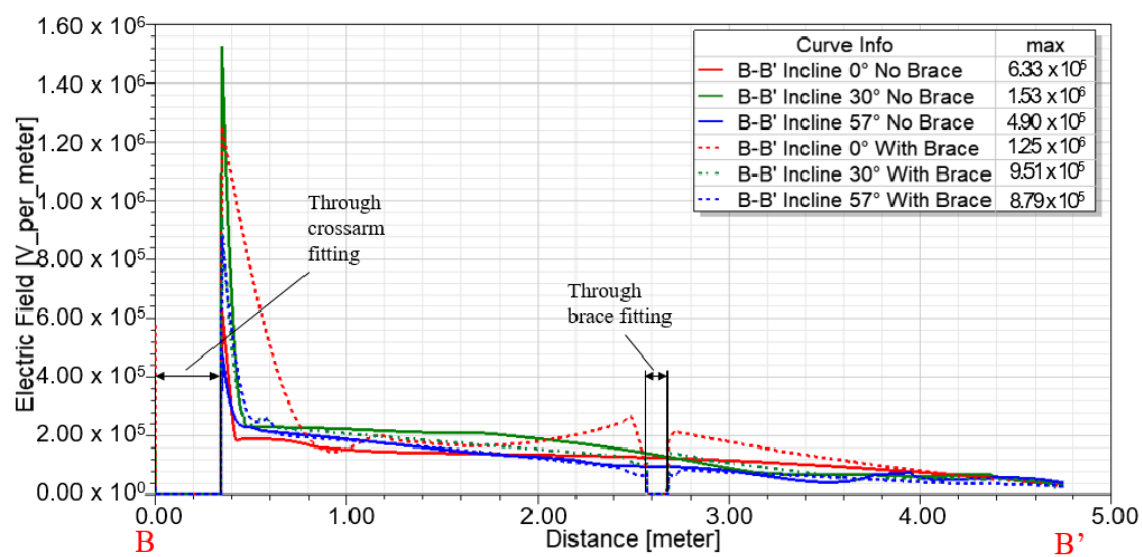

Figure 19. E-Field strength (measured at B-B') due to different swing angles.

At the main member $\left(\mathrm{A}-\mathrm{A}^{\prime}\right)$, the swing angle proportionally changed the profile of E-Field. According to Figure 20, significant changes in the E-Field magnitude can be found within the area of brace fitting and LV end of the crossarm (labeled as A), at which the maximum E-Field can be found. Without braces, changes of the swing angle to $30^{\circ}$ and $57^{\circ}$ caused an upsurge of E-Field by up to $183.4 \%$ and $270 \%$, respectively. However, the case with braces installed shows smaller increases of E-Field at the specified angle, with values of $17.8 \%$ and $121.3 \%$, correspondingly.

Referring to Table 4, the maximum E-Field values measured along the overall FRP crossarm structure and across the air were summarized. In general, the maximum E-Field on the FRP surface was found to be reduced when the swing angle was larger, but the opposite was true across the air. However, for the case without a brace, no clear trend was obtained, except for the fact that the largest maximum E-Field could be found when the swing angle was about $30^{\circ}$.

\subsection{Consideration of the Lightning Impulse Voltage Caused by Shielding Failure}

Figure 21 shows the difference of the potential distribution between both models applied with BIL and SF. It can be seen that the voltage intensity in different parts of the model was increased, causing a high voltage gradient across the model. As a result, it can be predicted that the E-Field 
intensity across the considered structures may also increase. The maximum E-Field values obtained from the simulations are shown in Table 5. As indicated in the table, the E-Field strength was much greater compared to those under the applied BIL voltage. It should be noted that for the case with no brace installed, the maximum E-Field strength across the air exceeded the dielectric strength of air i.e., $3.00 \times 10^{6} \mathrm{~V} / \mathrm{m}$, which might promote the formation of streamer and flashover. When the brace was included, the maximum E-Field exceeding the dielectric strength of air could only be found at a swing angle greater than $30^{\circ}$. In the case of the SF event, the E-Field strength on the FRP surface should be a concern, as it can be as high as $3.30 \times 10^{6} \mathrm{~V} / \mathrm{m}$, which is believed to greatly affect the long-term ageing of the main member of the crossarm.

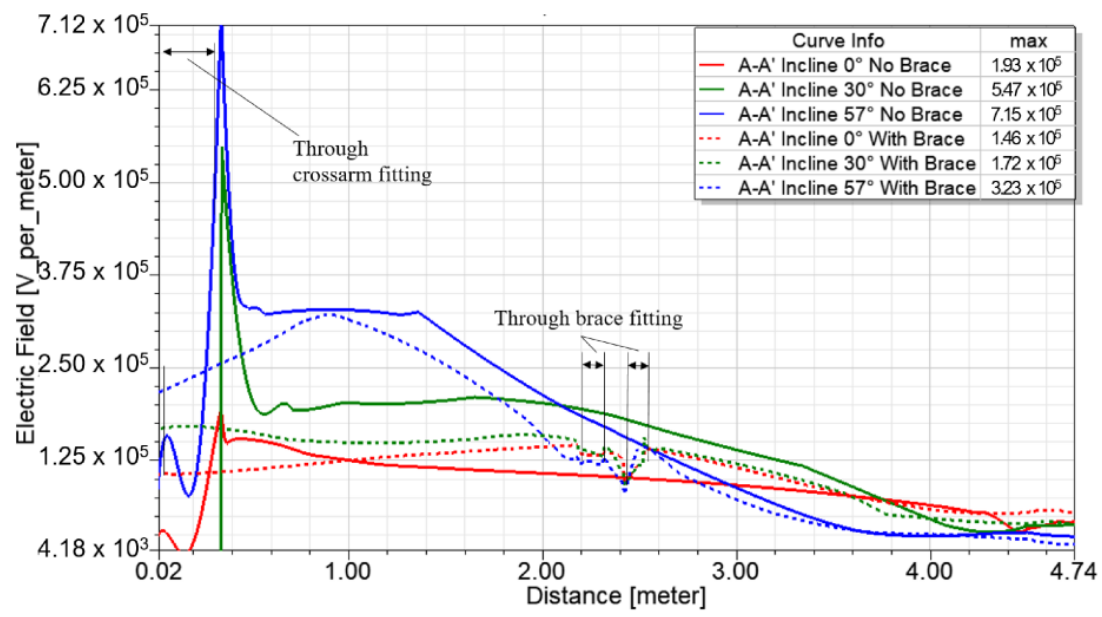

Figure 20. E-Field strength (measured at $\mathrm{A}-\mathrm{A}^{\prime}$ ) due to different swing angles.

Table 4. Maximum electric field under BIL voltage.

\begin{tabular}{ccccc}
\hline \multirow{2}{*}{ Applied LIV (kV) } & \multirow{2}{*}{ Brace } & \multirow{2}{*}{ Swing Angle } & \multicolumn{2}{c}{ Maximum E-Field (V/m) } \\
\cline { 3 - 5 } & & $0^{\circ}$ & On Overall FRP Surface & Across Air \\
\hline \multirow{3}{*}{1050} & No & $30^{\circ}$ & $6.33 \times 10^{5}$ & $1.74 \times 10^{6}$ \\
& & $57^{\circ}$ & $1.53 \times 10^{6}$ & $1.98 \times 10^{6}$ \\
& & $0^{\circ}$ & $7.15 \times 10^{5}$ & $1.68 \times 10^{6}$ \\
\cline { 3 - 5 } & & $30^{\circ}$ & $1.25 \times 10^{6}$ & $6.11 \times 10^{5}$ \\
& Yes & $57^{\circ}$ & $9.51 \times 10^{5}$ & $1.09 \times 10^{6}$ \\
& & $8.79 \times 10^{5}$ & $2.46 \times 10^{6}$ \\
\hline
\end{tabular}

Maximum allowable on FRP $=3.00 \times 10^{6}[10,11]$.
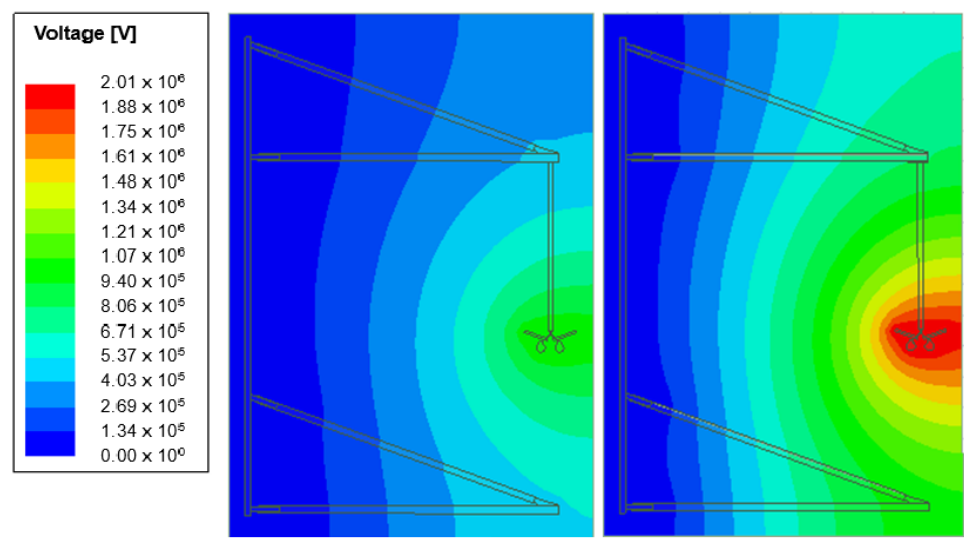

Figure 21. Comparison of the voltage distribution when subjected to both BIL (left) and SF (right) during the peak of the lightning impulse voltage (LIV) for the case without a brace. 
Table 5. Maximum electric field under SF voltage.

\begin{tabular}{ccccc}
\hline \multirow{2}{*}{ Applied LIV (kV) } & \multirow{2}{*}{ Brace } & Swing Angle & \multicolumn{2}{c}{ Maximum E-Field (V/m) } \\
\cline { 3 - 5 } & & $0^{\circ}$ & On Overall FRP Surface & Across Air \\
\hline \multirow{2}{*}{2000} & $30^{\circ}$ & $3.23 \times 10^{6}$ & $3.39 \times 10^{6}$ \\
& No & $57^{\circ}$ & $1.72 \times 10^{6}$ & $3.93 \times 10^{6}$ \\
& & $0^{\circ}$ & $2.41 \times 10^{6}$ & $3.24 \times 10^{6}$ \\
\cline { 3 - 5 } & \multirow{2}{*}{ Yes } & $30^{\circ}$ & $2.01 \times 10^{6}$ & $1.17 \times 10^{6}$ \\
& & $57^{\circ}$ & $1.80 \times 10^{6}$ & $4.16 \times 10^{6}$ \\
\hline
\end{tabular}

Maximum allowable on FRP $=3.00 \times 10^{6}[10,11]$.

\section{Conclusions}

In this paper, the effect of crossarm brace installation on the electrical performance of a $275 \mathrm{kV}$ FRP crossarm under an LIV condition was investigated. A quick estimation of CFO voltages was calculated across the high voltage phase conductor to the nearest earthed metallic part of the tower, namely, D1-D9. A simulation study was conducted to compare the performance of the crossarm before and after the brace installation, and the following conclusions can be drawn:

(1) The use of an FEM-based simulation has been proven to particularly locate the stresses along the composite FRP crossarm under a lightning impulse voltage. The 2D simulation sufficiently predicted the approximate value of the potential and E-Field distribution along the crossarm and in the surrounding air;

(2) The installation of the brace remarkably changed the potential distribution pattern of the surrounding area, which allowed a higher potential to shift closer to earthed parts, where the changes could obviously be seen in proximity of the crossarm. This alteration of the potential distribution is believed to cause a reduction of CFO across D1 and D7 of up to 4.9 and $10.6 \%$, respectively;

(3) The brace installation and insulator swing greatly influenced the maximum E-Field found on both the FRP surface and across the air. This study highlighted that the swing angle should be taken into consideration when designing a crossarm for a high voltage;

(4) By knowing the magnitude of the electric field on the crossarm structure, a proper mitigation plan can be considered to address the problem. The maximum E-Field strength on the FRP surface can be related to the cause of the insulation and physical degradation of the crossarm. It is important that the E-Field stress is kept at the minimum or at least below the dielectric strength of the material to ensure insulation integrity;

(5) The results presented in this study can further be enhanced by conducting a 3D simulation. It is worth mentioning that by producing a 3D model, the flashover distance will be more accurate and complexity of the structure can be introduced, thus providing accurate crossarm representation and more reliable results;

(6) Analyses conducted using FEM can provide a preliminary overview of the crossarm performance before the actual prototypes are produced. This can present more ideas for the designers to play around with in terms of the design, without investing so much in the production and testing the costly prototypes. Apart from being cost effective, this approach can save more time and is much more practical.

Author Contributions: Conceptualization, M.S.A.R.; resources, S.F.M.N.; writing—original draft preparation, M.S.A.R.; writing-review and editing, M.S.A.-R., M.O., and N.M.Z.; supervision, M.Z.A.A.K. All authors have read and agreed to the published version of the manuscript. 
Funding: The authors extend their sincerest appreciation and thanks to Universiti Tenaga Nasional for the guidance given and the current practices and knowledge shared. This work was conducted under the UNITEN BOLD Postgraduate Scholarship Programme.

Conflicts of Interest: The authors declare no conflict of interest.

\section{References}

1. Zhu, Y.; Wang, L.; Fang, J. Optimal insulation design for new-type transmission tower with composite crossarm. In Proceedings of the 2017 International Symposium on Electrical Insulating Materials (ISEIM), Toyohashi City, Japan, 11-15 September 2017; pp. 578-581.

2. Rawi, I.M.; Rahman, M.S.; Ab Kadir, M.Z.A.; Izadi, M. Wood and fiberglass crossarm performance against lightning strikes on transmission towers. In Proceedings of the International Conference on Power Systems Transient, Seoul, Korea, 26-29 June 2017; pp. 1-6.

3. Shao, J.; Wang, J.; Long, M.; Li, J.; Ma, Y. $5000 \mathrm{~h}$ multi-factor accelerated aging test of FRP made transmission tower: Characterization, thermal decomposition and reaction kinetics study. Polymers 2017, 9, 170.

4. Zhu, J.J.; Schoenoff, M.S. Effects of Natural Sunlight on Fiberglass Reinforced Polymers for Crossarms. In Proceedings of the 2018 IEEE Rural Electric Power Conference (REPC), Memphis, TN, USA, 6-9 May 2018; pp. 101-105.

5. Dai, J.; Jia, Y.; Fu, H.; Yang, J.; Xiao, X.; Liu, R. Investigation on the Aging Characteristics of FRP Composites Poles and Crossarms. In Proceedings of the 2018 China International Conference on Electricity Distribution (CICED), Tianjin, China, 17-19 September 2018.

6. Wang, Y.; Zhupanska, J. Lightning strike thermal damage model for glass fiber reinforced polymer matrix composites and its application to wind turbine blades. Compos. Struct. 2015, 132, 1182-1191.

7. Lu, T.; Solis-Ramos, E.; Yi, Y.; Kumosa, M. UV degradation model for polymers and polymer matrix composites. Polym. Degrad. Stab. 2018, 154, 203-210.

8. Beddu, S.; Syamsir, A.; Ishak, Z.A.M.; Yusof, Z.M.; Hudi, N.S.; Nabihah, S. Creep behavior of glass fibre reinforced polymer structures in crossarms transmission line towers. In Proceedings of the AIP Conference Proceedings 2031, Penang, Malaysia, 17-18 April 2018; p. 020039.

9. Sá, M.F.; Gomes, A.M.; Correia, J.R.; Silvestre, N. Creep behavior of pultruded GFRP elements-Part 1: Literature review and experimental study. Polym. Degrad. Stab. 2018, 154, 203-210.

10. Jahangiri, T.; Wang, Q.; Bak, C.L.; da Silva, F.F.; Skouboe, H. Electric stress computations for designing a novel unibody composite crossarm using finite element method. IEEE Trans. Dielectr. Electr. Insul. 2017, $24,3567-3577$.

11. Zachariades, C.; Rowland, S.M.; Cotton, I.; Peesapati, V.; Chambers, D. Development of electric field stress control device for a $132 \mathrm{kV}$ insulating crossarm using finite element analysis. IEEE Trans. Power Deliv. 2015, 31, 2105-2113.

12. Huang, D.; Ruan, J.; Chen, Y.; Huo, F.; Yu, S.; Liu, S. Calculation and measurement of potential and electric field distribution along $1000 \mathrm{kV}$ AC transmission line composite insulator. In Proceedings of the International Conference on Electrical Machines and Systems (ICEMS 2008), Wuhan, China, 17-20 October 2008; pp. 428-433.

13. IEC60383-2. Insulators for Overhead Lines with a Nominal Voltage Above 1000 V_Part 2: Definitions, Test Methods and Acceptance Criteria; International Electrotechnical Commission (IEC): Geneva, Switzerland, 1993.

14. Hileman, A.R. Insulation Coordination for Power Systems; CRC Press: Boca Raton, FL, USA, 2018.

15. Lings, R.J. EPRI AC Transmission Line Reference Book-200 kV and Above; Electric Power Research Institute: Washington, DC, USA, 2005.

16. Carlos, T.B.; Kaminski, J. Dynamic response due to cable rupture in a transmission lines guyed towers. Procedia Eng. 2017, 199, 116-121.

17. Bakar, M.S.A.; Mohamad, D.; Ishak, Z.A.M.; Yusof, Z.M.; Salwi, N. Durability control of moisture degradation in a transmission line towers. In Proceedings of the AIP Conference Proceedings 2031, Penang, Malaysia, 17-18 April 2018; p. 020027.

18. Parus, N. An Investigation into the Effects of Floating Objects on the Electrical Breakdown of Air Insulation under Steady State High Voltage Direct Current Conditions. Master's Thesis, University of the Witwatersrand, Johannesburg, South Africa, 2014. 
19. Caulker, D.; Ahmad, H.; Abdul-Malek, Z.; Yusof, S. Lightning overvoltage on an overhead transmission line during backflashover and shielding failure. In Proceedings of the 45th International Universities Power Engineering Conference (UPEC2010), Cardiff, UK, 31 August-3 September 2010; pp. 1-6.

20. Grzybowski, S.; Disyadej, T. Electrical performance of fiberglass crossarm in distribution and transmission lines. In Proceedings of the IEEE/PES Transmission and Distribution Conference and Exposition, Chicago, IL, USA, 21-24 April 2008; pp. 1-5.

21. CIGRE. Guide on Repair of conductors and Conductor-fitting System. CIGRE Tech. Broch. 2015, 708, 1-106.

Publisher's Note: MDPI stays neutral with regard to jurisdictional claims in published maps and institutional affiliations.

(C) 2020 by the authors. Licensee MDPI, Basel, Switzerland. This article is an open access article distributed under the terms and conditions of the Creative Commons Attribution (CC BY) license (http://creativecommons.org/licenses/by/4.0/). 